\title{
REVIEW
}

\section{Guselkumab for the Treatment of Psoriasis: A Review of Phase III Trials}

Mio Nakamura $\cdot$ Katherine Lee $\cdot$ Caleb Jeon · Sahil Sekhon •

Ladan Afifi - Di Yan · Kristina Lee - Tina Bhutani

Received: May 16, 2017 / Published online: June 21, 2017

(C) The Author(s) 2017. This article is an open access publication

\section{ABSTRACT}

Introduction: Interleukin (IL)-23 inhibitors are a new class of biologics currently undergoing clinical trials for the treatment of moderate-to-severe psoriasis. Phase III studies of guselkumab, an IL-23 receptor monoclonal antibody, are currently underway.

Methods: We summarize the available phase III results to date, establishing the efficacy and safety of guselkumab in patients with moderate-to-severe plaque psoriasis.

Results: Currently, there are available data of up to 48 weeks from two Phase III, multicenter, randomized, double-blind, placebo- and comparator-controlled clinical trials, VOYAGE 1 and VOYAGE 2. At week 16, the proportion of patients attaining at least a 90\% improvement from baseline in the Psoriasis Area and Severity Index (PASI 90) was 73.3\% in VOYAGE 1 and $70.0 \%$ in VOYAGE 2. Guselkumab remained

Enhanced content To view enhanced content for this article go to http://www.medengine.com/Redeem/ FC98F06027D19002.

M. Nakamura ( $₫)$ - C. Jeon · S. Sekhon · L. Afifi ·

D. Yan $\cdot$ K. Lee $\cdot$ T. Bhutani

Department of Dermatology, University of

California San Francisco, San Francisco, CA, USA

e-mail: Mio.Nakamura@ucsf.edu

K. Lee

University of California Los Angeles, Los Angeles, CA, USA efficacious through 48 weeks of treatment. Guselkumab maintained a satisfactory safety profile with the most frequently reported adverse events being nasopharyngitis, headache, and upper respiratory tract infection.

Conclusion: Phase III trials of Guselkumab suggest a favorable efficacy and safety profile of this novel drug. Although further studies are needed to assess long-term safety and efficacy, based on the results to date, guselkumab appears to be a promising therapeutic option for moderate-to-severe plaque-type psoriasis.

Keywords: Anti-interleukin-23; Biologics; Guselkumab; Phase III; Psoriasis; VOYAGE

\section{INTRODUCTION}

Psoriasis is a chronic inflammatory skin disease that affects approximately $3 \%$ of the adult US population [1]. The disease is thought to be a systemic inflammatory process and is associated with comorbidities such as psoriatic arthritis, cardiometabolic diseases, gastrointestinal diseases, kidney disease, malignancy, infection, and mood disorders [2]. Psoriasis can have a significant negative impact on quality of life, including impairment in physical and mental functioning, psychological well-being, and work productivity [3-5]. Safe and effective long-term treatment options for psoriasis are needed, not only for improving physical 
appearance but also for restoring quality of life and potentially decreasing the risk of comorbid diseases [6, 7].

There has been rapid development of novel psoriasis therapies over the past two decades, especially biologic agents including tumor necrosis factor (TNF)- $\alpha$ inhibitors, interleukin (IL)-12/23 inhibitors, and IL-17 inhibitors. A new class of biologics currently undergoing clinical trials includes the IL-23 inhibitors guselkumab, tildrakizumab, and risankizumab [8]. IL-23 is primarily produced by antigen-presenting cells and induces and maintains differentiation of T-helper (Th)-17 and Th-22 cells, which produce pro-inflammatory cytokines such as IL-17 and IL-22 that mediate the inflammation and epidermal hyperplasia of psoriasis [9]. IL-23 is composed of p19 and p40 subunits that bind to IL-23 receptor (IL-23R) and IL-12 receptor b1 (IL-12Rb1), which results in activation of pro-inflammatory Janus kinase 2 (JAK2), tyrosine kinase 2 (TYK2), and signal transducer and activator of transcription (STAT) signaling molecules [10].

Guselkumab (CNTO1959; Janssen Research \& Development, Spring House, PA, USA) is a fully human IgG1 lambda monoclonal antibody that binds to the p19 subunit of IL-23. Phase I and II studies have shown promising safety and efficacy in the treatment of moderate-to-severe psoriasis $[11,12]$. In the following article, the available results to date of the Phase III clinical trials establishing the efficacy and safety of guselkumab in patients with moderate-to-severe plaque psoriasis will be reviewed.

\section{METHODS}

We reviewed all published results of the Phase III clinical trials for guselkumab, which we identified by performing an English language literature search using PubMed with the following search terms: "guselkumab and psoriasis" and "IL-23 and psoriasis". References of relevant articles were hand-searched to identify other applicable publications. This article is based on published data from previously conducted studies and does not involve any new studies of human or animal subjects performed by any of the authors.

\section{RESULTS}

Data from two Phase III, multicenter, randomized, double-blind, placebo- and comparatorcontrolled clinical trials, VOYAGE 1 and VOYAGE 2 (ClinicalTrials.gov identifiers: NCT02207231 and NCT02207231, respectively) have been published to date. Up to 48 weeks of data are included. Both studies evaluated the efficacy and safety of guselkumab compared to placebo and adalimumab in patients with moderate-to-severe psoriasis [13, 14]. VOYAGE 2 also included a randomized withdrawal and retreatment period to evaluate the effect of interrupted treatment on the safety and efficacy of guselkumab.

The primary and secondary endpoints for VOYAGE 1 and VOYAGE 2 are shown in Table 1. Table 2 shows baseline demographic information of the subjects from each trial. Efficacy data of guselkumab compared to placebo and adalimumab from both trials are shown in Tables 3 and 4.

\section{VOYAGE 1}

\section{Study Design}

VOYAGE 1 was a phase III, randomized, double-blind, placebo- and active comparator (adalimumab)-controlled trial conducted at 101 global sites from December 2014 to April 2016. The study comprised a placebo-controlled period (weeks 0-16), after which patients taking placebo crossed over to receive guselkumab through week 48 . During the active-comparator period (weeks 0-48), guselkumab was compared with adalimumab. Therefore, all subjects were randomized to one of three treatment arms: (1) Guselkumab $100 \mathrm{mg}$ at weeks 0, 4, 12, and every 8 weeks through week 44 ; (2) placebo at weeks 0,4 , and 12 followed by guselkumab $100 \mathrm{mg}$ at weeks 16 and 20, and every 8 weeks through week 44; and (3) adalimumab $80 \mathrm{mg}$ at week 0 , $40 \mathrm{mg}$ at week 1 , and $40 \mathrm{mg}$ every 2 weeks through week 47 . Placebo was administered when required to maintain the blind.

Co-primary end points were the proportions of patients achieving an Investigator Global 
Table 1 Primary and secondary endpoints in VOYAGE 1 and VOYAGE 2

\begin{tabular}{|c|c|c|c|c|c|}
\hline & \multicolumn{3}{|l|}{ VOYAGE 1} & \multicolumn{2}{|l|}{ VOYAGE 2} \\
\hline & Week 16 & Week 24 & Week 48 & Week 16 & Week 24 \\
\hline \multicolumn{6}{|c|}{ Primary endpoints } \\
\hline IGA $0 / 1$ & Gus vs. Pla & & & Gus vs. Pla & \\
\hline PASI-90 & Gus vs. Pla & & & Gus vs. Pla & \\
\hline \multicolumn{6}{|c|}{ Secondary endpoints } \\
\hline IGA 0 & & Gus vs. Ada & Gus vs. Ada & & Gus vs. Ada \\
\hline IGA $0 / 1$ & Gus vs. Ada & Gus vs. Ada & Gus vs. Ada & Gus vs. Ada & Gus vs. Ada \\
\hline PASI-90 & Gus vs. Ada & Gus vs. Ada & Gus vs. Ada & Gus vs. Ada & Gus vs. Ada \\
\hline PASI-75 & Gus vs. Ada & & & Gus vs. Ada & \\
\hline ss-IGA $0 / 1$ & Gus vs. Pla & & & Gus vs. Pla & \\
\hline DLQI & Gus vs. Pla & & & Gus vs. Pla & \\
\hline PSSD & Gus vs. Pla & & & Gus vs. Pla & \\
\hline PSSD 0 & & Gus vs. Ada & & & Gus vs. Ada \\
\hline PASI-90 loss & & & & & Maintenance vs. withdrawal \\
\hline
\end{tabular}

Gus guselkumab, Pla placebo, $A d a$ adalimumab, $I G A$ investigator global assessment, $P A S I$ psoriasis area and severity index, $s s-I G A$ scalp-specific investigator global assessment, $D L Q I$ dermatology life quality index, $P S S D$ psoriasis symptoms and signs diary

Assessment (IGA) score of cleared/minimal disease (IGA 0/1) and 90\% or greater improvement in the Psoriasis Area and Severity Index (PASI) score from baseline (PASI 90) at week 16 in the guselkumab group versus placebo. Table 1 shows the major secondary endpoints, which include other efficacy measures (i.e. scalp) and patient-reported outcomes (i.e. psoriasis symptoms, health-related quality of life) in the guselkumab group versus placebo and adalimumab groups.

\section{Efficacy}

Guselkumab was superior to placebo and adalimumab for the co-primary end points and all major secondary end points, respectively (all $P<0.001) \quad$ (Tables 3, 4; Fig. 1a-c). Compared with placebo, significantly higher proportions of subjects taking guselkumab achieved IGA 0/1 (6.9\% vs. $85.1 \%)$ and PASI 90 (2.9\% vs. $73.3 \%)$ at week 16. Similarly, significantly higher proportions of subjects in the guselkumab versus adalimumab group achieved IGA 0/1 (85.1\% vs. $65.9 \%)$, PASI 90 (73.3\% vs. $49.7 \%)$, and PASI 75 $(91.2 \%$ vs. $73.1 \%)$ at week 16 . These findings were maintained through weeks 24 and 48 . The proportion of patients in the guselkumab group achieving scalp-specific IGA (ss-IGA) of $0 / 1$ was significantly higher versus placebo at week 16 and versus adalimumab at weeks 24 and 48 (all $P<0.001)$. Improvement in dermatology life quality index (DLQI) and Psoriasis Symptoms and Signs Diary (PSSD) scores were superior in the guselkumab group compared to placebo at week 16 and adalimumab at weeks 24 and 48 (all $P<0.001$ ) (Table 4).

\section{Safety}

During the placebo-controlled period (weeks $0-16)$, the proportions of patients with at least 
Table 2 Baseline demographics and clinical characteristics for all patients enrolled in VOYAGE 1 and VOYAGE 2

\begin{tabular}{|c|c|c|c|c|c|c|}
\hline & \multicolumn{3}{|l|}{ VOYAGE 1} & \multicolumn{3}{|l|}{ VOYAGE 2} \\
\hline & Placebo & Guselkumab & $\overline{\text { Adalimumab }}$ & Placebo & Guselkumab & $\overline{\text { Adalimumab }}$ \\
\hline Randomized patients, $n$ & 174 & 329 & 334 & 248 & 496 & 248 \\
\hline \multicolumn{7}{|l|}{ Age, years } \\
\hline Mean \pm SD & $44.9 \pm 12.90$ & $43.9 \pm 12.74$ & $42.9 \pm 12.58$ & $43.3 \pm 12.4$ & $43.7 \pm 12.2$ & $43.2 \pm 11.9$ \\
\hline Men, n (\%) & $119(68.4)$ & $240(72.9)$ & $249(74.6)$ & $173(69.8)$ & $349(70.4)$ & $170(68.5)$ \\
\hline \multicolumn{7}{|l|}{ Race, n (\%) } \\
\hline White & $145(83.3)$ & $262(79.6)$ & $277(82.9)$ & $206(83.1)$ & $408(82.3)$ & $200(80.6)$ \\
\hline Asian & $23(13.2)$ & $51(15.5)$ & $47(14.1)$ & $27(10.9)$ & $72(14.5)$ & $37(14.9)$ \\
\hline Black & $3(1.7)$ & $6(1.8)$ & $8(2.4)$ & $8(3.2)$ & $6(1.2)$ & $5(2.0)$ \\
\hline \multicolumn{7}{|l|}{ BMI, $\mathrm{kg} / \mathrm{m}^{2}$} \\
\hline Mean \pm SD & $28.9 \pm 6.89$ & $29.7 \pm 6.22$ & $29.8 \pm 6.48$ & $29.6 \pm 6.6$ & $29.6 \pm 6.5$ & $29.6 \pm 6.6$ \\
\hline \multicolumn{7}{|l|}{ Duration of psoriasis, years } \\
\hline Mean \pm SD & $17.6 \pm 12.44$ & $17.9 \pm 12.27$ & $17.0 \pm 11.27$ & $17.9 \pm 11.9$ & $17.9 \pm 12.0$ & $17.6 \pm 11.7$ \\
\hline \multicolumn{7}{|c|}{ Body surface area involvement, \% } \\
\hline Mean \pm SD & $25.8 \pm 15.93$ & $28.3 \pm 17.10$ & $28.6 \pm 16.66$ & $28.0 \pm 16.5$ & $28.5 \pm 16.4$ & $29.1 \pm 16.7$ \\
\hline \multicolumn{7}{|l|}{ IGA score, $0-4, \mathrm{n}(\%)$} \\
\hline Mild, 2 & 0 & 0 & $3(0.9)$ & $0(0.0)$ & $1(0.2)$ & $0(0.0)$ \\
\hline Moderate, 3 & $131(75.3)$ & $252(76.6)$ & $241(72.2)$ & $191(77.0)$ & $380(76.6)$ & $195(78.6)$ \\
\hline Severe, 4 & $43(24.7)$ & $77(23.4)$ & $90(26.9)$ & $57(23.0)$ & $115(23.2)$ & $53(21.4)$ \\
\hline \multicolumn{7}{|l|}{ PASI score, $0-72$} \\
\hline Mean $\pm S D$ & $20.4 \pm 8.74$ & $22.1 \pm 9.49$ & $22.4 \pm 8.97$ & $21.5 \pm 8.0$ & $21.9 \pm 8.8$ & $21.7 \pm 9.0$ \\
\hline Psoriatic arthritis, n (\%) & $30(17.2)$ & $64(19.5)$ & $62(18.6)$ & $46(18.5)$ & $89(17.9)$ & $44(17.7)$ \\
\hline \multicolumn{7}{|l|}{ Prior treatments, $\mathrm{n}(\%)$} \\
\hline Topical agents & $154(88.5)$ & $299(90.9)$ & $309(92.8)$ & $233(94.0)$ & $477(96.2)$ & $237(96.0)$ \\
\hline Phototherapy & $86(49.4)$ & $188(57.3)$ & $180(53.9)$ & $137(55.2)$ & $293(59.1)$ & $135(54.7)$ \\
\hline $\begin{array}{l}\text { Conventional systemic } \\
\text { agents }\end{array}$ & $92(52.9)$ & $210(63.8)$ & $215(64.4)$ & $149(60.1)$ & $331(66.7)$ & $159(64.1)$ \\
\hline Biologic agents & $34(19.5)$ & $71(21.6)$ & $70(21.0)$ & $54(21.8)$ & $101(20.4)$ & $49(19.8)$ \\
\hline
\end{tabular}

$B M I$ body mass index, $I G A$ investigator global assessment, $P A S I$ psoriasis area and severity index 


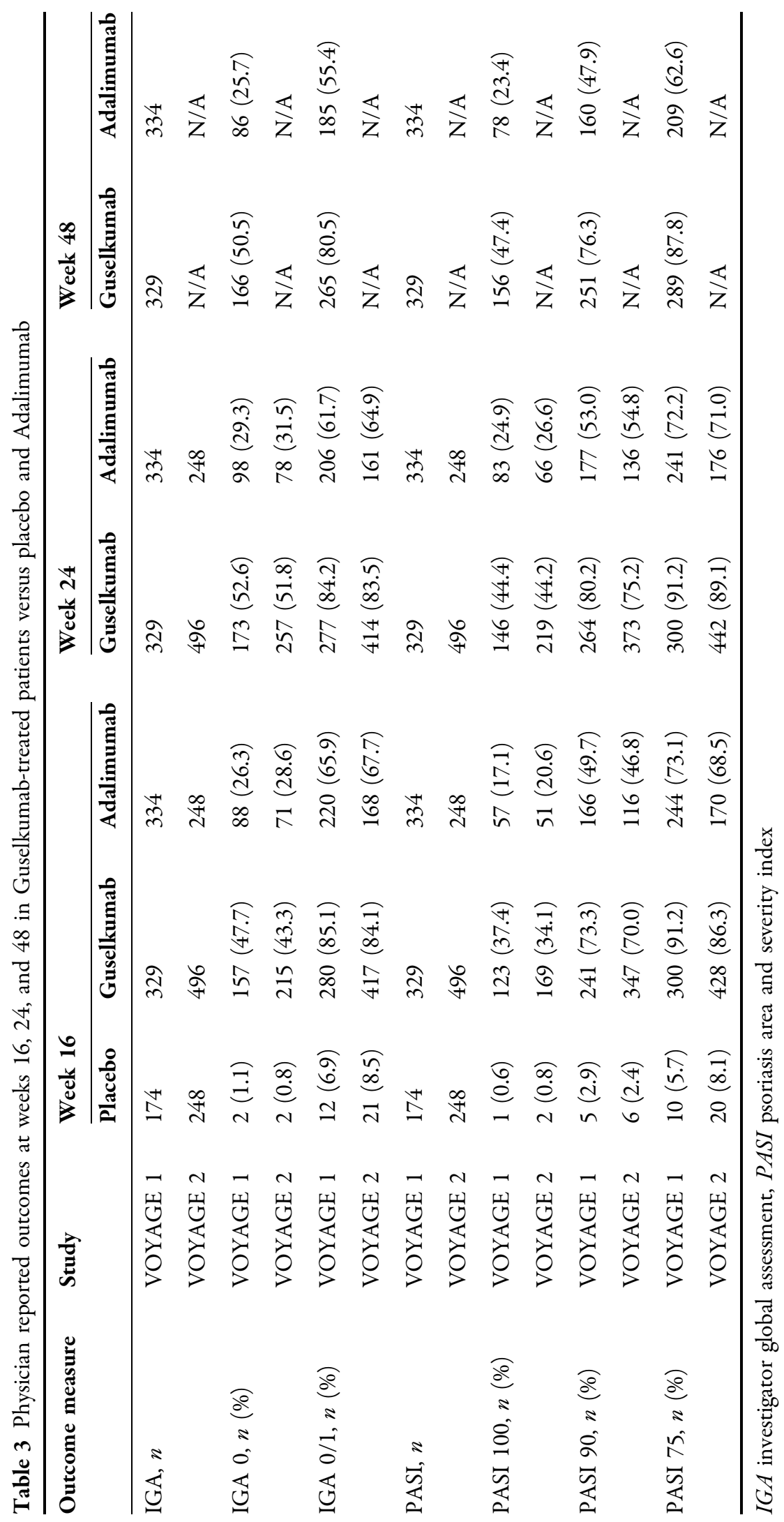




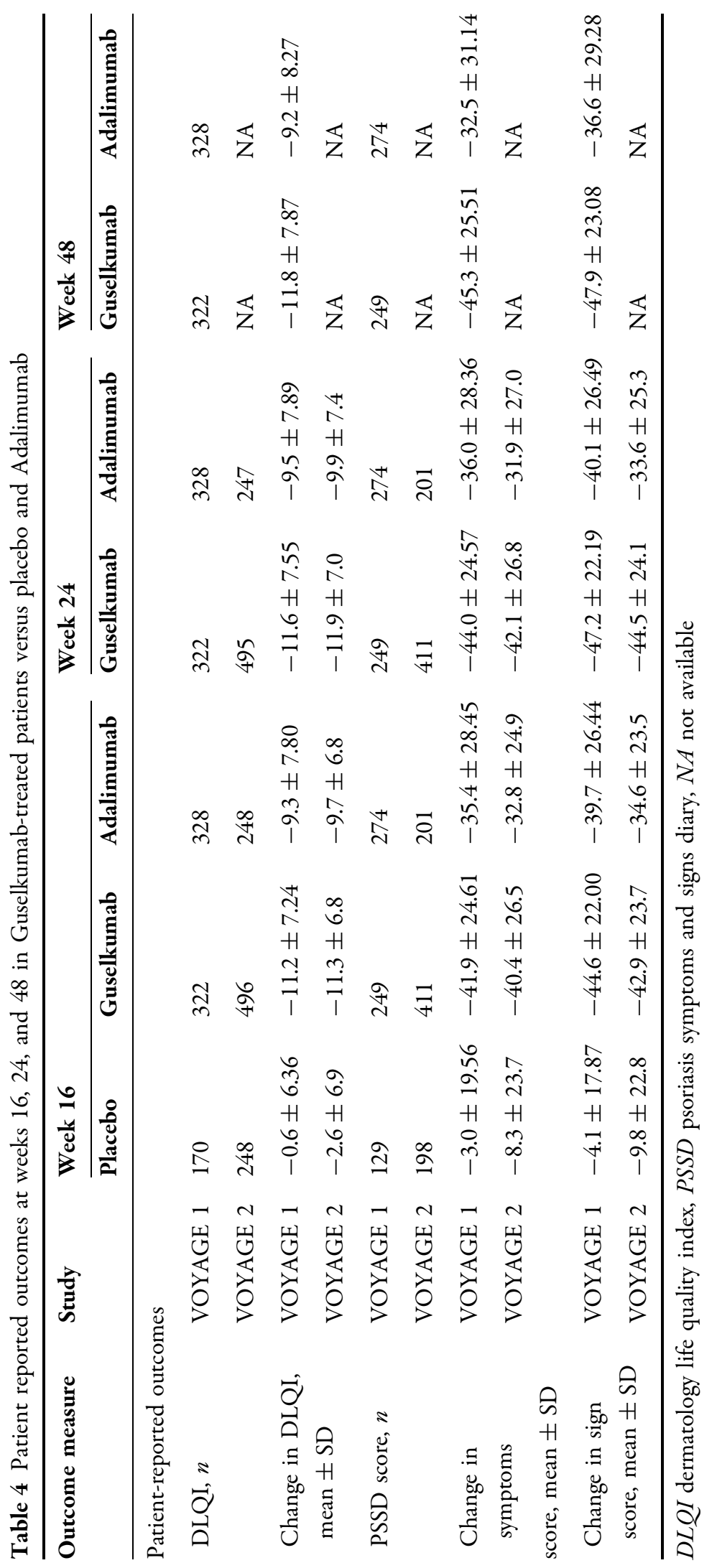



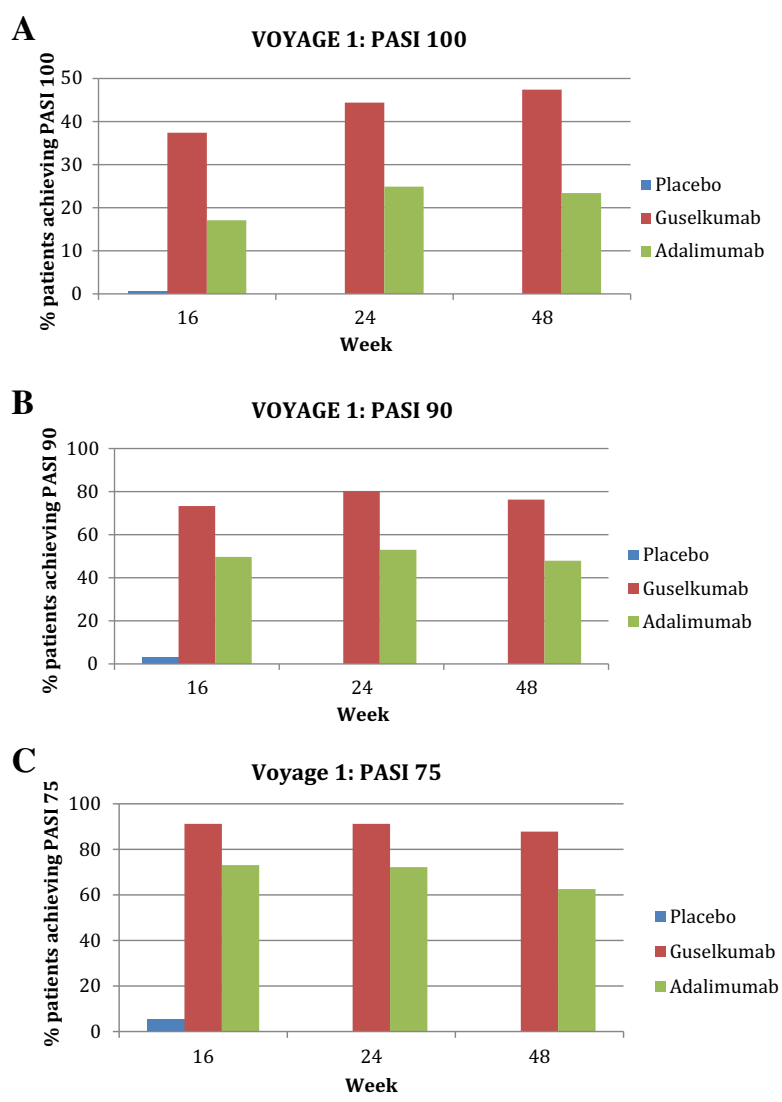

Fig. 1 Efficacy data from VOYAGE 1 showing percentage of patients achieving PASI 100 (a), PASI 90 (b), and PASI $75(\mathbf{c})$

one adverse event (AE), serious AEs and AEs leading to study agent discontinuation were comparable across treatment groups. The most commonly reported events were nasopharyngitis and upper respiratory tract infection. Rates of overall infections and infections requiring antibiotic treatment were comparable across treatment. Two patients in the adalimumab group experienced serious infections (both cellulitis). Only one malignancy was reported, which was a nonmelanoma skin cancer (NMSC) [basal cell carcinoma (BCC)] in the guselkumab group. One myocardial infarction [major adverse cardiovascular event (MACE)] occurred in each of the guselkumab and adalimumab groups through week 16 .

Similarly, through week 48 , proportions of patients with at least one $\mathrm{AE}$, an $\mathrm{AE}$ leading to discontinuation, or a serious $\mathrm{AE}$ were similar in the guselkumab and adalimumab groups. Rates of serious infections were equal in both the guselkumab and adalimumab groups (two reports each). Overall infections and infections requiring antibiotic treatment occurred at comparable rates across treatment groups. There were two additional NMSCs (1 BCC each in the guselkumab and adalimumab groups) and two malignancies (prostate and breast in the guselkumab group). Through week 48 , the rates of injection site reaction (ISR) were lower in the guselkumab group compared to adalimumab (2.2\% vs. 9.0\%). Antibodies to guselkumab were detected in 26 of 492 patients (5.3\%) through week 44. Titers were generally low (81\% $\leq 1: 320$ ), and no association was observed between antibody development and reduced efficacy or ISR occurrence.

\section{VOYAGE 2}

\section{Study Design}

VOYAGE 2 was a phase III, multicenter, randomized, double blind, placebo- and active comparator (adalimumab)-controlled study conducted at 115 global sites from November 2014 to May 2016. The study comprised a placebo-controlled period during weeks $0-16$, an active comparator-controlled period during weeks $0-28$, and a randomized withdrawal and retreatment period during weeks 28-72. Subjects were first randomized to one of three treatment arms: (1). Guselkumab $100 \mathrm{mg}$ at weeks $0,4,12$, and 20; (2). Placebo at weeks 0, 4, and 12, then guselkumab at weeks 16 and 20; (3). Adalimumab $80 \mathrm{mg}$ at week $0,40 \mathrm{mg}$ at week 1 , and every 2 weeks thereafter through week 23. At week 28, guselkumab-treated subjects achieving PASI 90 (responders) were re-randomized in a 1:1 ratio to guselkumab or placebo. For those re-randomized to placebo, upon loss of $50 \%$ or more of week 28 PASI response, subjects were retreated with guselkumab $100 \mathrm{mg}$ at weeks 0,4 , and every 8 weeks thereafter. Guselkumab non-responders continued guselkumab treatment. While placebo to guselkumab non-responders at week 28 continued guselkumab every 8 weeks, placebo to 
guselkumab responders received placebo every 8 weeks beginning at week 28 . Upon loss of $50 \%$ or more of week 28 PASI response, patients were retreated with guselkumab $100 \mathrm{mg}$ at weeks 0 , 4 , and every 8 weeks thereafter. Adalimumab non-responders initiated guselkumab at week $28,100 \mathrm{mg}$ at weeks 0,4 , and every 8 weeks thereafter. Adalimumab responders received placebo, and upon loss of $50 \%$ or more of week 28 PASI response, they were given guselkumab $100 \mathrm{mg}$ at weeks 0,4 , and every 8 weeks thereafter. Placebo was administered when required to maintain the blind.

Co-primary end points were the proportions of patients achieving an IGA 0/1 and PASI 90 at week 16 in the guselkumab group compared with placebo. Table 2 shows major secondary endpoints including other efficacy measures (i.e. scalp) and patient reported outcomes (i.e. psoriasis symptoms, health-related quality of life) in guselkumab versus placebo and adalimumab groups. The time to loss of PASI 90 response was compared between maintenance versus withdrawal groups during weeks $28-48$.

\section{Efficacy}

Guselkumab was superior to placebo and adalimumab for all co-primary end points and major secondary end points, respectively (all $P<0.001$ ) (Tables 3, 4; Fig. 2a-c). Compared to placebo, significantly higher proportions of subjects taking guselkumab achieved IGA 0/1 (8.5\% vs. $84.1 \%)$ and PASI 90 (2.4\% vs. $70.0 \%)$ at week 16 . Similarly, significantly higher proportion of subjects in the guselkumab versus adalimumab group achieved IGA 0/1 (84.1\% vs. 67.7), PASI 90 (70.0\% vs. $46.8 \%)$, and PASI 75 (86.3\% vs. $68.5 \%)$ at week 16 . The proportion of patients in the guselkumab group achieving ss-IGA 0/1 was significantly higher compared to placebo at week 16 and adalimumab at weeks 16 and 24 (all $P<0.001$ ). Improvement in DLQI and PSSD scores from baseline were superior in the guselkumab group compared to placebo at week 16 and adalimumab at weeks 24 and 48 (all $P<0.001$ ) (Table 4).

In the randomized withdrawal and re-treatment phase, guselkumab week 28 responders
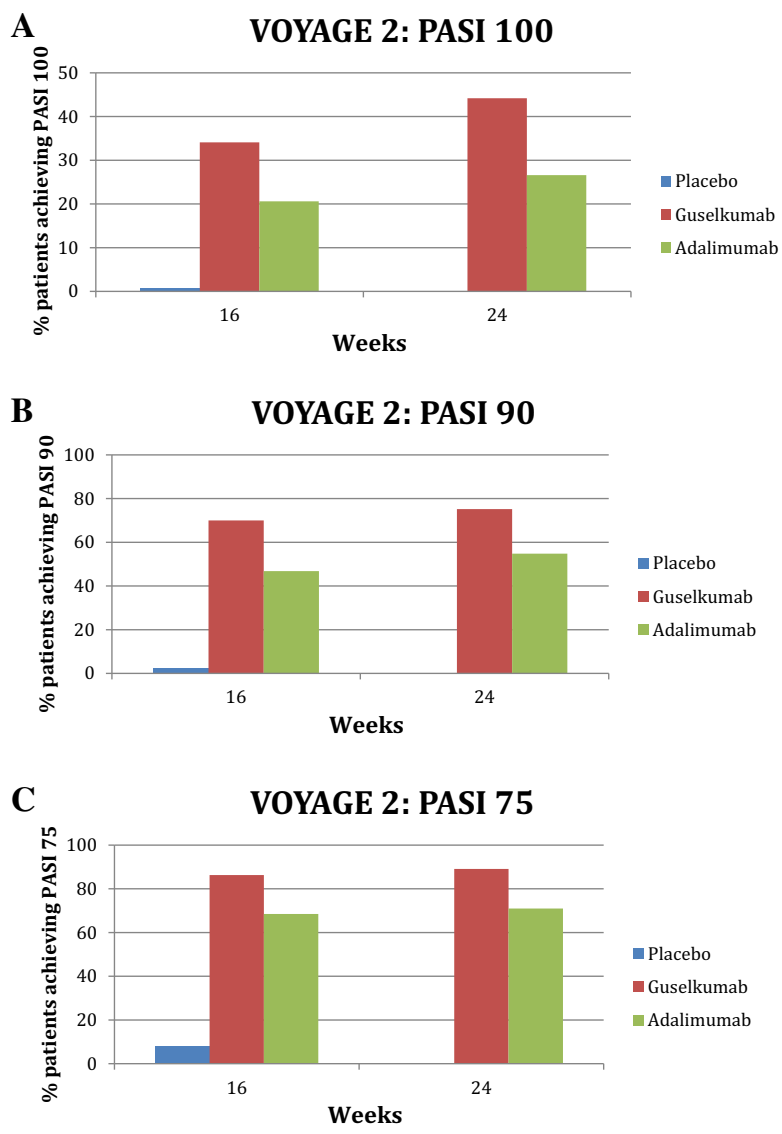

Fig. 2 Efficacy data from VOYAGE 2 showing percentage of patients achieving PASI 100 (a), PASI 90 (b), and PASI $75(\mathbf{c})$

continuing guselkumab (maintenance group) were more likely to maintain PASI 90 responses versus responders re-randomized to placebo (withdrawal group). For patients in the withdrawal group, the median time to loss of PASI 90 response was 15.2 weeks ( 23 weeks after last guselkumab dose). Among patients withdrawn from guselkumab at week 28, PASI 90 response rates began to worsen compared to the maintenance group at week 32 . Through week 48 , PASI 90 was maintained in $88.6 \%$ of patients in the maintenance group versus $36.8 \%$ of those in the withdrawal group. At week 48, clinical responses (IGA/PASI) were significantly greater in the maintenance than in the withdrawal group $(P<0.001)$. Improvements in DLQI and PSSD from baseline were also significantly greater at week 48 in the maintenance versus 
withdrawal groups $(P<0.001)$. In the 112 adalimumab non-responders who initiated guselkumab at week 28 (5 weeks after the last adalimumab dose), PASI 90 and PASI 100 rates increased from baseline after switching, reaching 66.1 and $28.6 \%$, respectively, at week 48 .

\section{Safety}

During the placebo-controlled period (weeks $0-16)$, the proportions of patients with one or more AEs, AEs leading to discontinuation, and SAEs were comparable between the guselkumab and placebo groups. The most commonly reported events were nasopharyngitis, headache, and upper respiratory tract infections. The rates of infection were similar among groups. There were no reports of malignancy through week 16. A higher proportion of adalimumab patients had ISR versus guselkumab patients $(6.9 \%$ vs. $2.6 \%)$. One MACE (myocardial infarction) occurred in the adalimumab group.

During the active comparator period (weeks $0-28)$, the proportions of patients with 1 or more $\mathrm{AE}$, AEs leading to discontinuation, and SAEs were comparable between the guselkumab and adalimumab groups. Infections and infections requiring treatment were also comparable between guselkumab and adalimumab groups. Serious infections were reported in equal number in the guselkumab (bronchitis, erysipelas, and soft-tissue infection) and adalimumab [two cases of tuberculosis (1 disseminated) and 1 injection-site abscess] groups. All patients with a history or symptoms of active tuberculosis were excluded from the study, and no details of the individual cases of tuberculosis are publicly available at this time. One malignancy (prostate cancer) and two NMSC [1 squamous cell carcinoma (SCC) in the guselkumab group and 1 BCC in the placebo-guselkumab group] were reported. There were two cases of MACE (1 myocardial infarction each in the guselkumab and adalimumab groups).

During the randomized withdrawal and retreatment period (weeks 28-48), there was one SAE of appendicitis. Through week 48, there were two additional reports of NMSC (one BCC and 1 SCC) and one additional report of
MACE in the placebo-guselkumab group. Antibodies to guselkumab were detected in 57 of 869 patients $(6.6 \%)$ through week 48 . Titers were generally low $(88 \% \leq 1: 160)$ and there were no apparent associations observed between antibody development and decreased efficacy or ISR development.

\section{DISCUSSION}

Results from the examined Phase III clinical trials, VOYAGE 1 and VOYAGE 2, demonstrate the safety and efficacy of guselkumab in the treatment of moderate-to-severe plaque psoriasis in a diverse group of patients. At week 16, the proportions of patients achieving IGA $0 / 1$ and PASI-90 were statistically significantly superior compared to placebo. Guselkumab's efficacy as measured by IGA 0, IGA 0/1, PASI-90, and PASI-75 was also superior to adalimumab at weeks 12, 24, and 48. Guselkumab also showed effectiveness in treating difficult-to-treat areas including the scalp (ss-IGA), nails (f-PGA, NAPSI), hands and feet (hf-PGA). Patient reported outcomes and health-related quality of life (DLQI, PSSD) also improved and mirrored improvement in investigator-reported psoriasis measures.

The randomized withdrawal and re-treatment study in VOYAGE 2 showed that maintenance treatment with guselkumab (100 mg every 8 weeks) is superior in maintaining PASI 90 response compared to interrupted therapy. This suggests that IL-23 inhibition does not cause long-lasting remission of psoriasis. The median time to loss of PASI 90 response after discontinuing guselkumab therapy was 15.2 weeks. Guselkumab was also effective in patients who failed to respond to adalimumab.

Although head-to-head studies are lacking, compared to all biologic agents currently available for the treatment of moderate-to-severe psoriasis, Phase III studies of guselkumab report some of the highest PASI-90 rates (at week 16, $73.3 \%$ in VOYAGE 1 and $70.0 \%$ in VOYAGE 2) to date [15]. Recent studies have shown that patients who reach PASI 90 or PASI 100 experience greater improvement in quality of life compared to those who achieve PASI 75 [16]. 
The potential of guselkumab to achieve PASI 90 in $70 \%$ or more of patients suggests that guselkumab may have a significant positive impact on patient quality of life. Furthermore, patients maintain PASI 90 response for a median of 15.2 weeks (106.4 days) after discontinuing guselkumab. This is significantly greater than the time to relapse (defined as PGA $\geq 3$ ) for etanercept $50 \mathrm{mg}$ BIW of 72 days [17] and etanercept $50 \mathrm{mg}$ QW of 36.9 days [18]. The median time to relapse (defined as PGA $\geq 3$ ) for adalimumab $40 \mathrm{mg}$ QOW is 141 days [19]. The time to loss of PASI 90 is not reported. Regardless, the development of this unique, highly effective therapy provides optimism for patients whose psoriasis remains refractory to currently available biologic agents and adds to the growing armamentarium of psoriasis therapies.

The high efficacy rate of guselkumab may be related to the mechanism of IL-23 inhibition. IL-23 is thought to induce production of TNF- $\alpha$, IL-17A, and IL-22, which are all implicated in the pathogenesis of psoriasis [20-22]. Targeting IL-23, a key cytokine driving various other effector cytokines, may be responsible for the high efficacy and durable responses up to week 48. Further long-term studies are needed to understand the long-term efficacy of guselkumab.

Overall, guselkumab appears to have a favorable safety profile: Phase III trials have found the most common AEs to include nasopharyngitis, headache, and upper respiratory tract infection. Serious infection, malignancy, and MACE do not appear to be increased in patients treated with guselkumab compared to placebo and adalimumab. ISR were more common in patients treated with adalimumab compared to guselkumab. There were five cases of NMSC, four of which were in the guselkumab group (two BCCs and three SCCs) and the other in the adalimumab group. A recent study has shown an increased risk of NMSC in psoriasis patients treated with TNF- $\alpha$ inhibitors [23]. However, other studies have suggested that psoriasis-related factors such as treatment with phototherapy and chronic inflammatory dysfunction of psoriasis, not biologic therapies, may be factors responsible for the observed increased risk of NMSC in psoriasis patients [24]. The details of the individual cases of NMSC in VOYAGE 1 and 2 are not publicly available to date; therefore, it is unknown whether or not these particular patients have a history of treatment with phototherapy. Guselkumab antibody formation was observed in a small percentage of patients $(5.3 \%$ in VOYAGE 1 , $6.6 \%$ in VOYAGE 2) but did not have any observable effect on the efficacy or safety of guselkumab. The clinical implications of these findings are unknown, and further long-term studies are necessary to determine AEs resulting from long duration of treatment with guselkumab in a larger population of patients. The favorable AE profile of guselkumab observed in Phase III studies suggest that IL-23 may be a more psoriasis-specific cytokine compared to other cytokines such as TNF- $\alpha$ and IL-17 [25].

\section{CONCLUSIONS}

Guselkumab has generated encouraging data for efficacy and safety in the treatment of moderate-to-severe chronic plaque psoriasis. The Phase III clinical trials results suggest that guselkumab is superior to placebo at week 16 and is better able to clear or almost clear psoriasis plaques compared to adalimumab. The effects are maintained through week 48 . Further long-term studies are required to validate the promising long-term efficacy and safety profile of guselkumab.

\section{ACKNOWLEDGEMENTS}

No funding or sponsorship was received for this study or publication of this article. All named authors meet the International Committee of Medical Journal Editors (ICMJE) criteria for authorship for this manuscript, take responsibility for the integrity of the work as a whole, and have given final approval for the version to be published.

Disclosures. Tina Bhutani conducts research for Abbvie, Janssen, Leo Pharma, Mela, and Merck. Mio Nakamura, Katherine Lee, Caleb Jeon, Sahil Sekhon, Ladan Afifi, Di Yan, and Kristina Lee have nothing to disclose. 
Compliance with Ethics Guidelines. This article is based on previously conducted studies and does not involve any new studies of human or animal subjects performed by any of the authors.

Data Availability. Data sharing is not applicable to this article as no datasets were generated or analyzed during the current study.

Open Access. This article is distributed under the terms of the Creative Commons Attribution-NonCommercial 4.0 International License (http://creativecommons.org/licenses/ by-nc/4.0/), which permits any noncommercial use, distribution, and reproduction in any medium, provided you give appropriate credit to the original author(s) and the source, provide a link to the Creative Commons license, and indicate if changes were made.

\section{REFERENCES}

1. Rachakonda T, Schupp C, Armstrong A. Psoriasis prevalence among adults in the United States. J Am Acad Dermatol. 2014;70(3):512-6.

2. Takeshita J, Grewal S, Langan SM, et al. Psoriasis and comorbid diseases: epidemiology. J Am Acad Dermatol. 2017;76(3):377-90.

3. Rapp SR, Exum ML, Reboussin DM, Feldman SR, Fleischer A, Clark A. The physical, psychological and social impact of psoriasis. J Health Psychol. 1997;2(4):525-37.

4. Rapp SR, Feldman SR, Exum ML, Fleischer AB, Reboussin DM. Psoriasis causes as much disability as other major medical diseases. J Am Acad Dermatol. 1999;41(3 Pt 1):401-7.

5. Pearce DJ, Singh S, Balkrishnan R, Kulkarni A, Fleischer AB, Feldman SR. The negative impact of psoriasis on the workplace. J Dermatolog Treat. 2006;17(1):24-8.

6. Bhutani T, Patel T, Koo B, Nguyen T, Hong J, Koo J. A prospective, interventional assessment of psoriasis quality of life using a nonskin-specific validated instrument that allows comparison with other major medical conditions. J Am Acad Dermatol. 2013;69(2):e79-88.

7. Wu JJ, Guérin A, Sundaram M, Dea K, Cloutier M, Mulani P. Cardiovascular event risk assessment in psoriasis patients treated with tumor necrosis factor- $\alpha$ inhibitors versus methotrexate. J Am Acad Dermatol. 2017;76(1):81-90.

8. Dong J, Goldenberg G. New biologics in psoriasis: an update on IL-23 and IL-17 inhibitors. Cutis. 2017;99(2):123-7.

9. Puig L. The role of IL 23 in the treatment of psoriasis. Expert Rev Clin Immunol. 2017; . doi:10.1080/ 1744666X.2017.1292137.

10. Teng MW, Bowman EP, McElwee JJ, et al. IL-12 and IL-23 cytokines: from discovery to targeted therapies for immune-mediated inflammatory diseases. Nat Med. 2015;21(7):719-29.

11. Sofen H, Smith S, Matheson RT, et al. Guselkumab (an IL-23-specific mAb) demonstrates clinical and molecular response in patients with moderate-to-severe psoriasis. Allergy Clin Immunol. 2014;133(4):1032-40.

12. Gordon KB, Duffin KC, Bissonnette R, et al. A Phase 2 Trial of Guselkumab versus Adalimumab for Plaque Psoriasis. N Engl J Med. 2015;373(2):136-44.

13. Blauvelt A, Papp KA, Griffiths CE, et al. Efficacy and safety of guselkumab, an anti-interleukin-23 monoclonal antibody, compared with adalimumab for the continuous treatment of patients with moderate to severe psoriasis: results from the phase III, double-blinded, placebo- and active comparator-controlled VOYAGE 1 trial. J Am Acad Dermatol. $2017 ; 76(3): 405-17$.

14. Reich K, Armstrong AW, Foley P, et al. Efficacy and safety of guselkumab, an anti-interleukin-23 monoclonal antibody, compared with adalimumab for the treatment of patients with moderate to severe psoriasis with randomized withdrawal and retreatment: results from the phase III, double-blind, placebo- and active comparator-controlled VOYAGE 2 trial. J Am Acad Dermatol. 2017;76(3):418-31.

15. Farahnik B, Beroukhim $\mathrm{K}$, Nakamura $\mathrm{M}$, et al. Anti-IL-17 agents for psoriasis: a review of phase III data. J Drugs Dermatol. 2016;15(3):311-6.

16. Revicki DA, Willian MK, Menter A, Saurat J-HH, Harnam N, Kaul M. Relationship between clinical response to therapy and health-related quality of life outcomes in patients with moderate to severe plaque psoriasis. Dermatology (Basel). 2008;216(3):260-70.

17. Ortonne JP, Taieb A, Ormerod AD, et al. Patients with moderate-to-severe psoriasis recapture clinical response during re-treatment with etanercept. $\mathrm{Br} \mathrm{J}$ Dermatol. 2009;161:1190-5. 
18. Moore A, Gordon KB, Kang S, et al. A randomized, open-label trial of continuous versus interrupted etanercept therapy in the treatment of psoriasis. J Am Acad Dermatol. 2007;56:598-603.

19. Papp K, Crowley J, Ortonne JP, Leu J, Okun M, et al. Adalimumab for moderate to severe chronic plaque psoriasis: efficacy and safety of retreatment and disease recurrence following withdrawal from therapy. Br J Dermatol. 2011;164:434-41.

20. Reich K, Papp KA, Matheson RT, et al. Evidence that a neutrophil-keratinocyte crosstalk is an early target of IL-17A inhibition in psoriasis. Exp Dermatol. 2015;24:529-35.

21. Miossec P, Korn T, Kuchroo VK. Interleukin-17 and type 17 helper T cells. N Engl J Med. 2009;361:888-98.

22. Zheng $\mathrm{Y}$, Danilenko DM, Valdez $\mathrm{P}$, et al. Interleukin-22, a T(H) 17 cytokine, mediates IL-23-induced dermal inflammation and acanthosis. Nature. 2007;445:648-51.

23. Asgari MM, Ray GT, Geier JL, Quesenberry CP. Malignancy rates in a large cohort of patients with systemically treated psoriasis in a managed care population. J Am Acad Dermatol. 2017;76(4):632-8.

24. van Lümig PP, Menting SP, van den Reek JM, et al. An increased risk of non-melanoma skin cancer during TNF-inhibitor treatment in psoriasis patients compared to rheumatoid arthritis patients probably relates to disease-related factors. J Eur Acad Dermatol Venereol. 2015;29(4):752-60.

25. Blauvelt A, Lebwohl MG, Bissonnette R. IL-23/ IL-17A dysfunction phenotypes Inform possible clinical effects from anti-IL-17A therapies. J Investig Dermatol. 2015;135:1946-53. 